\title{
The Effect of White-Noise Mask Level on Sinewave Contrast Detection Thresholds and the Critical-Band-Masking Model
}

\author{
Ignacio Serrano-Pedraza ${ }^{1}$ and Vicente Sierra-Vázquez ${ }^{2}$ \\ ${ }^{1}$ University of Newcastle \\ ${ }^{2}$ Complutense University of Madrid
}

\begin{abstract}
It is known that visual noise added to sinusoidal gratings changes the typical U-shaped threshold curve which becomes flat in log-log scale for frequencies below 10c/deg when gratings are masked with white noise of high power spectral density level. These results have been explained using the critical-band-masking (CBM) model by supposing a visual filter-bank of constant relative bandwidth. However, some psychophysical and biological data support the idea of variable octave bandwidth. The CBM model has been used here to explain the progressive change of threshold curves with the noise mask level and to estimate the bandwidth of visual filters. Bayesian staircases were used in a 2IFC paradigm to measure contrast thresholds of horizontal sinusoidal gratings $(0.25-8 \mathrm{c} / \mathrm{deg})$ within a fixed Gaussian window and masked with one-dimensional, static, broadband white noise with each of five power density levels. Raw data showed that the contrast threshold curve progressively shifts upward and flattens out as the mask noise level increases. Theoretical thresholds from the CBM model were fitted simultaneously to the data at all five noise levels using visual filters with log-Gaussian gain functions. If we assume a fixed-channel detection model, the best fit was obtained when the octave bandwidth of visual filters decreases as a function of peak spatial frequency.
\end{abstract}

Keywords: contrast detection threshold, spatial white noise, critical-band-masking paradigm

El ruido visual añadido a enrejados sinusoidales cambia la típica forma en $U$ de la curva de umbral, que se transforma en una función casi uniforme (en escala log-log) cuando los enrejados son enmascarados por ruido blanco cuya densidad espectral de potencia (o nivel) es alta. Ese hecho se ha explicado mediante el modelo de enmascaramiento basado en bandas críticas (modelo CBM) suponiendo que la anchura de banda relativa (en octavas) de los filtros visuales es constante. Sin embargo, estudios biológicos y psicofísicos apoyan la idea de la variación de la anchura de banda con la frecuencia de sintonía de los filtros. En este trabajo se ha utilizado el modelo CBM para explicar el cambio progresivo de la curva de umbral con el nivel del ruido y, a la vez, para estimar la anchura de banda de los filtros visuales. Para ello, se midieron (utilizando escaleras bayesianas en un paradigma 2IFC) los umbrales de contraste de enrejados sinusoidales (de 0.25 a $8 \mathrm{c} / \mathrm{gav}$ ), presentados dentro de una ventana Gaussiana fija y enmascarados por ruido blanco 1D estático con cada uno de cinco niveles. Los resultados indican que, en efecto, al aumentar el nivel del ruido, los umbrales de contraste se hacen cada vez mayores y, a la vez, la curva de umbral se va aplanando progresivamente. Utilizando el modelo CBM, los umbrales teóricos se ajustaron a los datos simultáneamente en todos los niveles de ruido suponiendo que la función de ganancia de los filtros visuales es log-Gaussiana y que la detección se lleva a cabo por el filtro sintonizado a la frecuencia del enrejado. Con esos supuestos razonables, el ajuste fue adecuado sólo cuando la anchura de banda relativa de los filtros visuales decrece con su frecuencia espacial de sintonía.

Palabras clave: umbral de contraste, ruido blanco, modelo de enmascaramiento

This research was supported by grant \#BSO2002-00192 from the Ministerio de Ciencia y Tecnología (Spain) and grant \#PR145/0312169 from the Comunidad de Madrid.

We thank L. E. Lopez-Bascuas for his insightful comments on this work. We also thank M. A. García-Pérez for suggesting the numerical evaluation of Equation A1 in the Appendix, and M. D. Luna for her corrections to the manuscript. Preliminary results were presented at the $28^{\text {th }}$ European Conference on Visual Perception held in A Coruña, August 22-26, 2005, and reported in abstract form by Serrano-Pedraza and Sierra-Vázquez (2005).

Address correspondence to: Dr. V. Sierra Vázquez, Dpto. de Psicología Básica I. Facultad de Psicología. Universidad Complutense de Madrid, Campus de Somosaguas, 28223 Madrid (Spain). Tel: + 3491394 3139. E-mail: vicente@psi.ucm.es 
The ability to detect a stimulus embedded in noise tells us much about the properties of the human visual system. In fact, most of our knowledge about visual processing of spatial patterns has come from studies of detection of a target masked with visual noise, especially when targets are sinusoidal gratings. In many studies, the masked data have been explained using the so-called critical-band-masking (CBM) paradigm. According to this model, transferred from auditory psychophysics ${ }^{1}$ (Fletcher, 1940; Patterson, 1974, 1976) to visual psychophysics, the contrast detection threshold (CDT) of a sinusoidal waveform masked with noise is proportional to the amount of mask power leaking through the single filter involved in detection (Henning, Hertz, \& Hinton, 1981; Losada \& Mullen, 1995; Pelli, 1981; Solomon, 2000). Thus, masked CDT is a measure of the area of overlap between filter gain and noise spectrum, and, as a consequence, some channel modulation transfer function (MTF) characteristics (e.g., spectral shape and spatialfrequency bandwidth) could be inferred from the data relating CDT to noise parameters. Because we use this model to analyze our data, it will be described in detail in the following section.

A variety of noise masks have been used to study the spatial-frequency filters that detect gratings. The present paper examines the masking of sine waves by onedimensional (1D) static white noise. It is known that, without external noise, the sinusoidal grating threshold curve at photopic illuminance levels has a typical U shape when plotted on a log-log scale (DePalma \& Lowry, 1962; Van Nes \& Bouman, 1967). When white noise is added, CDT increases with the noise power spectral density or level $\left(N_{0}\right)$ (Pelli, 1981; Stromeyer \& Julesz, 1972; Thomas, 1985), except for an initial decrease due to stochastic resonance (Blackwell, 1998), at the same time as the U-shaped contrast threshold curve progressively shifts upward and flattens out (Blackwell, 1998). When gratings are masked with high-level 2D white noise, the threshold curve becomes completely flat (i.e., CDT is independent of spatial frequency), at least for spatial frequencies below $10 \mathrm{c} / \mathrm{deg}$ (Rovamo, Franssila, \& Näsänen, 1992; Schofield \& Georgeson, 1999). In summary, white noise added to sinusoidal gratings increases their CDTs and changes the shape of contrast detection curves.

These results have been explained by means of the CBM model assuming filters with a constant relative bandwidth (in octaves) (Blackwell, 1998; Rovamo et al., 1992). However, biological (De Valois, Albrecht, \& Thorell, 1982; De Valois \& De Valois, 1988) and psychophysical (Schofield \& Georgeson, 2003; Solomon,
2000; Wilson, McFarlane, \& Phillips, 1983) data suggest that visual filter bandwidth decreases as a function of spatial frequency tuning of the filter. These disagreements in the spatial vision literature about the variation of octave bandwidth with filter peak frequency make it necessary to disentangle the relationship between these two filter characteristics.

The effect of white noise level on CDTs has been studied by varying the noise level for a limited number of spatial frequencies (Losada \& Mullen, 1995; Pelli, 1981; Stromeyer \& Julesz, 1972; Thomas, 1985) or by varying the spatial frequencies for a limited number of white noise levels (Blackwell, 1998; Rovamo et al., 1992). Obviously, in these cases, data showed only partially the change of threshold detection curve with noise level. In an intensive study, Solomon (2000) obtained masking patterns with 1D static white noise, and low-pass and high-pass filtered noise for a sufficient number of spatial frequencies and noise levels. As a result, he found that filter octave bandwidth decreases with filter peak frequency when detection is mediated either by the channel tuned to the frequency of the stimulus (the fixed-channel model) or by the channel that maximizes the signal-to-noise ratio (the best-channel model). Solomon's stimuli were sinusoidal gratings windowed by a two-dimensional (2D) circular Gaussian weighting function whose size was proportional to the period of the test grating, but he deliberately ignored this fact in computations. Regrettably, in this case, the grating contrast power of the Gabor patch is dependent on window size, as we show in the Appendix, and, consequently, ignoring the Gaussian window in computations may affect inferences. On the contrary, it can be shown that the contrast power of a Gabor patch with a fixed Gaussian window is independent of window size for the spatial frequencies used, thus allowing us to ignore it in computations without practical consequences.

The aims of this work are to apply the CBM model to explain the change of shape of threshold curves with noise level and to examine whether the filter bandwidth variation with peak spatial frequency can be confirmed when using Gabor patches with a fixed, 2D Gaussian window as signals. In order to show the progressive change of the threshold curves as noise level increases, we measured CDTs for sinusoidal grating of six spatial frequencies in the presence of 1D static white noise at five levels. Then, we applied the CBM model to fit the variation patterns of the threshold detection curves with noise level and estimate the relationship between the octave bandwidth and the peak spatial frequency of the hypothetical visual filters involved in detection.

\footnotetext{
1 The critical-band-masking model is referred to in the literature on hearing as the "power spectrum model" of masking (Moore, 1997).
} 


\section{The Critical-Band-Masking Paradigm}

\section{The Critical Band Masking Model}

The CBM model makes three main assumptions (Moore, 1997): (a) Stimuli are processed by a bank of linear bandpass filters; (b) the observer uses the stimulus energy passing through the single filter involved in detection; and (c) the CDT of the stimulus corresponds to a constant signal-to-noise ratio at the output of the filter. In its visual version, the CBM model predicts that the squared CDT of a sinusoidal grating (with spatial frequency $u_{0}$ ) used as signal test and masked with visual $1 \mathrm{D}$ noise of power spectrum $\rho, m^{2}\left(u_{0}\right)$, is

$$
m^{2}\left(u_{0}\right)=\frac{m_{0}^{2}\left(\xi_{k}\right)+\frac{4}{s} \int_{0}^{+\infty} \rho(u)\left|H\left(u ; \xi_{k}\right)\right|^{2} \mathrm{~d} u}{\left.\mid H\left(u_{0}\right) ; \xi_{k}\right)\left.\right|^{2}},
$$

where $\xi_{k}$ is the peak spatial frequency of the channel that detects the signal, $m_{0}\left(\xi_{k}\right)$ is the CDT of a grating of spatial frequency $\xi_{k}$ without external noise, $s$ is the sensitivity parameter, and $\left|H\left(u ; \xi_{k}\right)\right|$ is the MTF of the channel centered at spatial frequency $\xi_{k}$ responsible for detection. (Note: $\left|H\left(u ; \xi_{k}\right)\right|^{2}$ is the filter gain function). Equation 1, which is quoted in many studies, is derived in Serrano-Pedraza (2005). (For a derivation of a similar equation, with minor differences, see Solomon, 2000, his Equations 4 and 5). Note that, for simplicity, Equation 1 is for sinusoidal gratings without spatial windowing.

Below, for quantitative predictions, we choose as contrast sensitivity function (CSF; i.e., the reciprocal of $m_{0}$ ) the following expression:

$$
C S(u)=A u \exp [-a u]
$$

(Kelly, 1975), and we adopt the popular log-Gaussian function $^{2}$ as the MTF of visual filter (Morrone \& Burr, 1988):

$$
\left|H\left(u ; \xi_{i}\right)\right|= \begin{cases}\exp \left[-\frac{\operatorname{In}^{2}\left(|u| \xi_{i}\right)}{2 \alpha^{2}\left(\xi_{i}\right)}\right] & \Leftrightarrow \mathrm{u} \neq 0 \\ 0 & \Leftrightarrow \mathrm{u}=0,\end{cases}
$$

where $\xi_{i}$ is the peak spatial frequency of filter and is the spatial spread of filter $i$. The relative bandwidth (full-width at half height), in octaves, is obtained from. We adopt two forms for the relationship between $B_{o c t}\left(\xi_{i}\right)=\frac{2 \sqrt{2}}{\sqrt{\ln 2}} \times \alpha\left(\xi_{i}\right)$ and peak spatial frequency: an exponential decreasing function of peak spatial frequency and a constant function. The first form is

$$
B_{\text {oct }}\left(\xi_{i}\right)=b+B \exp \left(-\xi_{i}\right), \quad \mathrm{b} \geq 0, \quad B \geq 0
$$

While Equation 4 maintains a decreasing relationship, its advantage over a logarithmic decrease (Schofield \& Georgeson, 2003; Solomon, 2000) is that it prevents either zero or negative values for relative bandwidth from being obtained. However, if $B=0$ in Equation $4, B_{o c t}$ will be constant, therefore, in our fits, we forced $B_{o c t}$ to be constant, that is,

$$
B_{o c t}\left(\xi_{i}\right)=b
$$

\section{The Fixed-Channel Detection Model}

When an interpretative model of data is chosen, estimates of filter bandwidth depend on the detection model proposed (Henning et al., 1981). It has been shown that white noise prevents (theoretically) the off-frequency looking phenomenon, that is, the fact that the stimulus may not be detected by the channel tuned to its nominal frequency (Serrano-Pedraza, 2005; Serrano-Pedraza \& Sierra-Vázquez, 2006). Thus, we use as detection model the above mentioned fixed-channel model (Solomon, 2000), or a detection model in which sinusoidal signals of spatial frequency $u_{0}$ are always detected by the channel centered at the spatial frequency of the signal (i.e., $\xi_{k}=u_{0}$ ). White noise has the same power spectral density at all spatial frequencies (i.e.p $(u)=N_{0}$ ), so that Equation 1 becomes

$$
m^{2}\left(u_{0} ; N_{0}\right)=m_{0}^{2}\left(u_{0}\right)+N_{0} \frac{4}{s} \int_{0}^{+\infty}\left|H\left(u ; u_{0}\right)\right|^{2} \mathrm{~d} u,
$$

(see Patterson, 1974, 1976, with minor differences), where $m\left(u_{0} ; N_{0}\right)$ is the CDT of masked Gabor patch of $u_{0} \mathrm{c} / \mathrm{deg}$ at noise level $N_{0}$. Provided that the absolute threshold is low compared with that of the noise level, Equation 6 indicates that masked (squared) CDT is proportional to the power of effective noise transmitted by the filter involved in detection (as required by the third assumption above). More specifically, if the sensitivity is constant, it follows that, at every spatial frequency, the threshold elevation must be proportional to the noise level, where the theoretical estimated value of proportionality constant for $u_{0}$ is the product of $4 / s$ and the area under the gain curve of channel $u_{0}, Q\left(u_{0}\right)$,

$$
Q\left(u_{0}\right)=\sqrt{\pi} \alpha_{0} \exp \left[\frac{1}{4} \alpha^{2}\left(u_{0}\right)\right] u_{0},
$$

\footnotetext{
2 Some authors (Majaj, Pelli, Kurshan, \& Palomares, 2002; Solomon \& Pelli, 1994; Talgar, Pelli, \& Carrasco, 2004) adopt a quadratic function of $\log$ spatial frequency as $\log$ gain function of filters. The difference with Equation 3 is immaterial because it is a trivial issue to show that a log-Gaussian function plotted on a log-log scale is a quadratic function.
} 
where $\alpha\left(u_{0}\right)$ is the spatial spread of filter centered at $\alpha\left(u_{0}\right)=\frac{\sqrt{\ln 2}}{2 \sqrt{2}} \times B_{o c t}\left(u_{0}\right)$, with (Because the visual filter bandwidth is lower than the band of noise, to calculate the integral, we ignored the limits of the pseudo-white noise band, with no loss of generality. Nevertheless, in data fits, the real noise band limits are maintained). Thus, the CBM model with white noise maskers, for a fixed spatial frequency, predicts a linear relationship between squared CTD and noise level whose proportionality constant equals to $(4 / \mathrm{s}) Q_{0}\left(\mathrm{u}_{0}\right)$, that is,

$$
m^{2}\left(u_{0} ; N_{0}\right)=m_{0}^{2}\left(u_{0}\right)+N_{0} \frac{4}{s} Q\left(u_{0}\right) N_{0},
$$

\section{Method}

\section{Observers}

The first author (IS) and one paid, experienced psychophysical observer (GB), who was unaware of the purpose of the experiment, took part in the study. Both observers had normal visual acuity and viewed the screen binocularly with natural pupils.

\section{Stimulus Construction}

The stimuli were either 1D gratings embedded in 1D white noise (test signal plus mask) or 1D white noise alone (mask). Both the test signal and the mask were static. They can be described by Equation 9 .

$$
L(x, y)=L_{0}\left(1+m_{T E S T} f(x, y)+m_{M A S K} n(x, y)\right)
$$

where $L(x, y)$ is luminance at location $(x, y), L_{0}$ is mean luminance in $\mathrm{cd} / \mathrm{m}^{2}, f$ is the sinusoidal test signal, $m_{T E S T}$ is the Michelson contrast of the test, $n$ is the white noise, and $m_{\text {MASK }}$ is the noise masker contrast parameter that controls the noise power spectral density. When only noise mask was presented, $m_{T E S T}$ was set to zero; otherwise it was changed according to Bayesian staircases (see later in Procedure). All stimuli were horizontally oriented to prevent monitor artifacts when spatial frequency was relatively high (García-Pérez \& Peli, 2001). We used as test signals sinusoidal gratings of six spatial frequencies $\left(u_{0}=0.25,0.5\right.$, $1,2,4$, and $8 \mathrm{c} / \mathrm{deg}$ ) spatially windowed by a fixed $2 \mathrm{D}$ Gaussian function or Gabor patch, that is,

$$
f(x, y)=\exp \left[-\frac{x^{2}}{2 s_{\mathrm{x}}^{2}}-\frac{y^{2}}{2 s_{\mathrm{y}}^{2}}\right] \cos \left(2 \pi u_{0} \mathrm{y}\right)
$$

where $s_{x}, s_{y}$, in deg, are the standard deviations of the Gaussian window $\left(s_{x}=s_{y}=2.5 \mathrm{deg}\right)$. As a masker we used noise samples of 1D static white noise (see a sample in Figure 1a), that is, noise nominally composed by all spatial frequencies and all of them with the same amplitude. In practice, masks were band-pass noise with a lower frequency limit at $0.125 \mathrm{c} / \mathrm{deg}$ and no component above $16 \mathrm{c} / \mathrm{deg}$. However, these pseudo-white noises can be considered white noise for the relevant visual filter under certain conditions (Losada \& Mullen, 1995) that were met for all of them. Pseudo-white noise samples were generated first in the spatial-frequency domain from a two-sided flat power spectral density function, $\rho(u)=N_{0}$ (Figure 1c), and a phase spectrum in which phase is a random variable uniformly distributed on $(-\pi, \pi]$ (Figure $1 \mathrm{~d})$. The results were then Fourier-transformed into the spatial domain. It is known that luminance levels of white noise have a Gaussian distribution (Green \& Swets, 1966; Legge, Kersten, \& Burgess, 1987) with standard deviation ${ }^{3} \sigma=\sqrt{2 W N_{0}} L_{\text {ave }}$, where $W$ is the one-sided spectral bandwidth of noise (in this paper, $W=15.875 \mathrm{c} / \mathrm{deg}), N_{0}$ is the noise power level, $L_{\text {ave }}=\sum \sum L_{\text {MASK }}(i, j) /(N R \times N C)$ is the average of the noise luminances, and $N R$ and $N C$ are the dimensions, in pixels, of the image. Formula for $\sigma$ follows easily from Legge et al. (1987, note 29), with $N_{0}=c_{R M S}^{2} / 2 W$ Figure $1 \mathrm{~b}$ shows the Gaussian luminance distribution of the white noise sample of Figure 1a. Six separate test signals (one per spatial frequency) and 140 white noise images (two per trial) were previously constructed using Matlab as digital images of $512 \times 512$ pixels and 256 gray levels and stored on a hard disk drive; thus, $f$ and $n$ of Equation 9 are really contrast functions relative to the digital images.

\section{Equipment and Display}

Digital images were presented on a high-resolution 19" monitor (Eizo Flexcan T765, Eizo Corp., Japan) in monochrome mode using a VSG2/3F Issue 4a graphics card (Cambridge Research Systems Ltd., UK) under the control of a computer (Pentium II-MMX, 400Mhz) that also stored the images. The monitor frame rate was $120 \mathrm{~Hz}(60 \mathrm{~Hz}$ per complete image when frame interleaving was used). The relationship between pixel value and screen luminance was measured by an OptiCal photometer (Cambridge Research

\footnotetext{
${ }^{3} \sigma$ is the standard deviation of the luminances, not the root-mean-square contrast, $c_{R M S}$, or standard deviation of the contrast function as in Losada and Mullen (1995). In fact, $c_{R M S}=\sigma / L_{\text {ave }}$ (Stromeyer \& Julesz, 1971).
} 


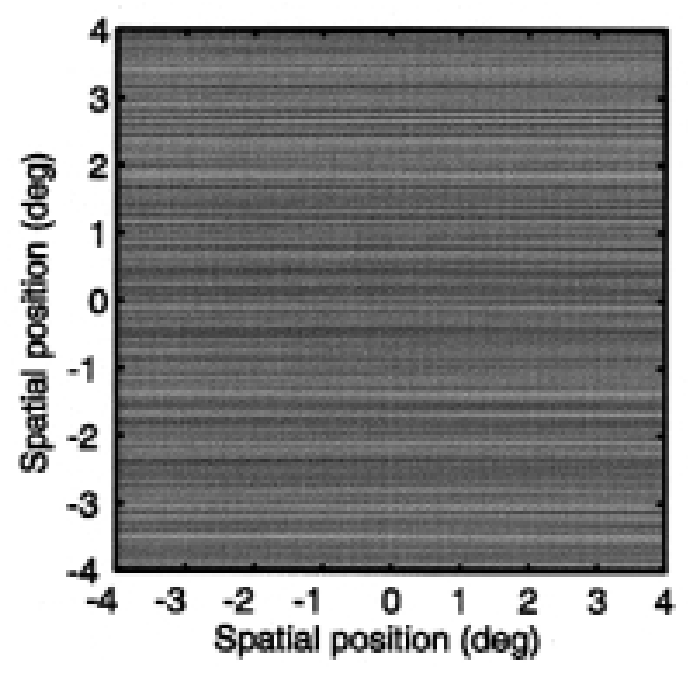

a

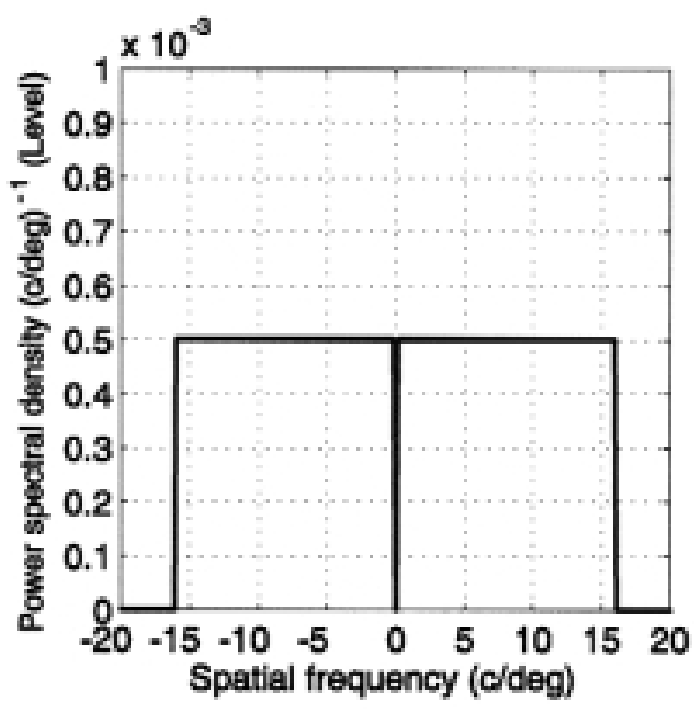

C

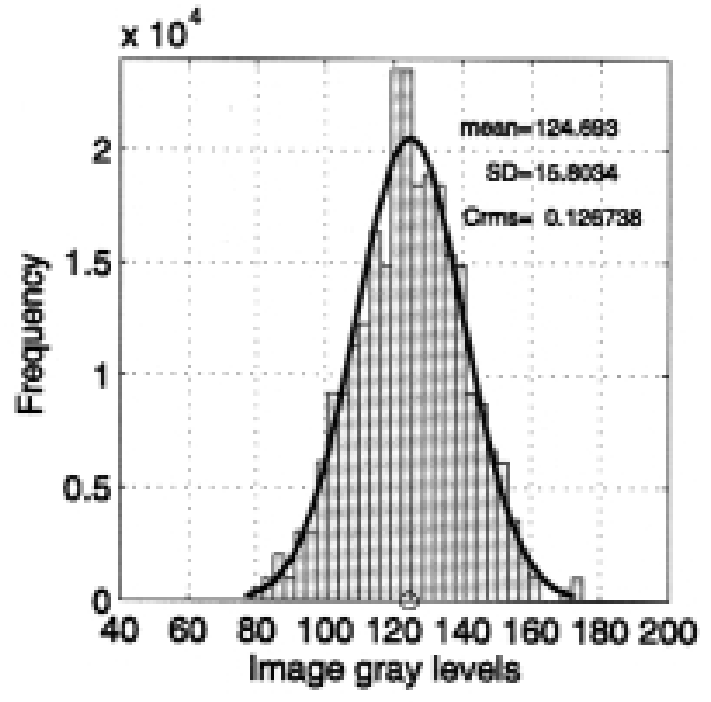

b

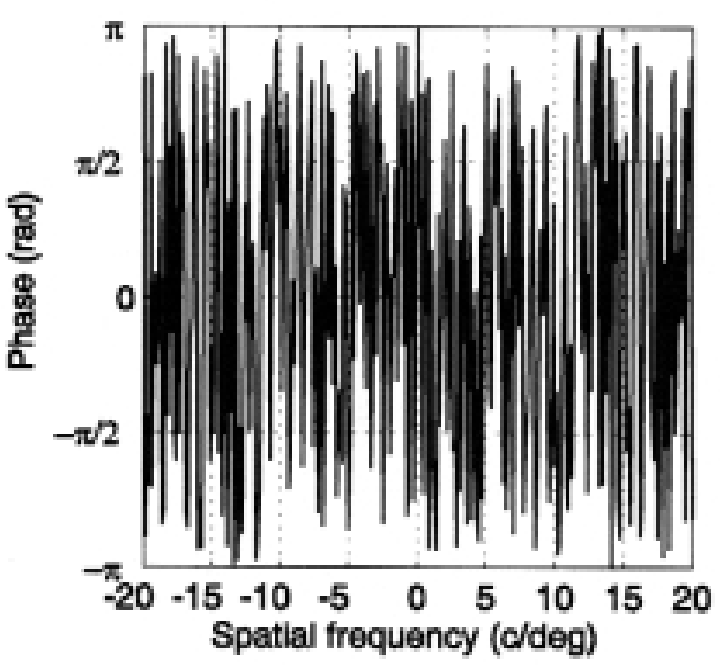

d

Figure 1. Sample of noise mask. (a) Image of 1D white noise. (b) Histogram of luminances and parameter values. (d) Plot of power spectral density. (e) Plot of phase spectrum.

Systems Ltd.) interfaced to the computer, and the monitor's gamma non-linearity was corrected using software look-up tables (LUT) in the VSG. This calibration also provided 15bit grayscale resolution. The calibration was verified every few weeks.

Each digital image was linearly converted into a displayed image via the LUT of the graphics card during each frame. This allowed both test and mask contrasts to be changed online without losing intensity levels and without changing the stored digital image (Schofield, 1998). The images were presented at the center of the screen in a square of $14.68 \mathrm{~cm}$ per side and the remainder of the screen was at mean luminance. They were viewed at a distance of $105 \mathrm{~cm}$ and subtended an area of $8 \times 8 \mathrm{deg}$. The mean luminance of the image area, $L_{0}$, was $15 \mathrm{~cd} / \mathrm{m}^{2}$, corresponding to value 128 of the image gray level.

\section{Masking Procedure}

We used the frame interleaving method to mask the test signal (Schofield \& Georgeson, 1999; Solomon, 2000). The advantage of this method is that the test contrast can be varied independently of the contrast of the noise mask in which the test is embedded (Schofield, 1998). Stimuli were displayed by presenting tests and masks separately in alternate frames of video sequences (images of Figure $2 a$ and $2 b$ ). 


\section{Frame 1 Test}

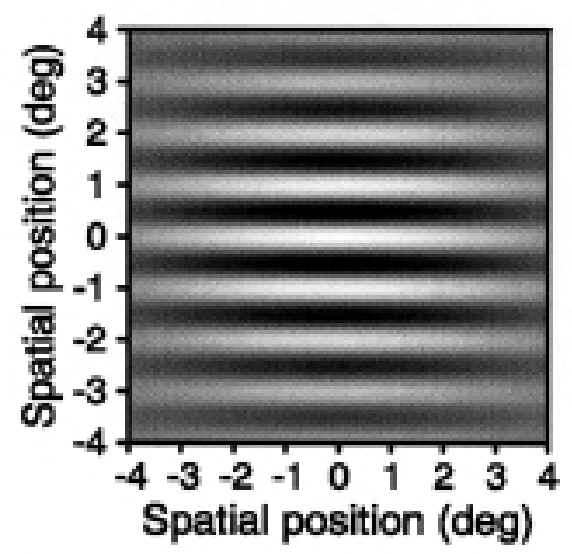

Frame 2 Mask

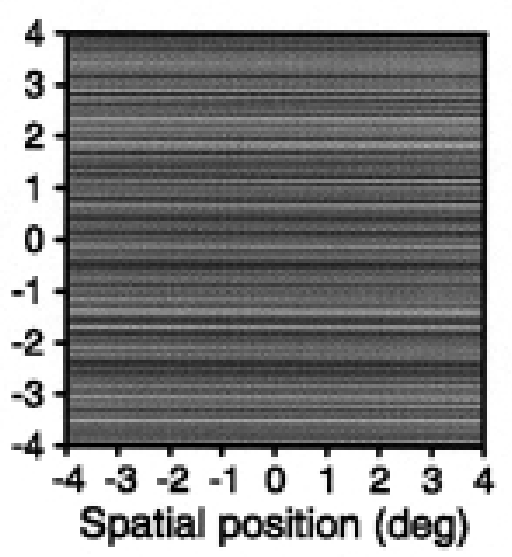

\section{Result of frame interleave procedure}

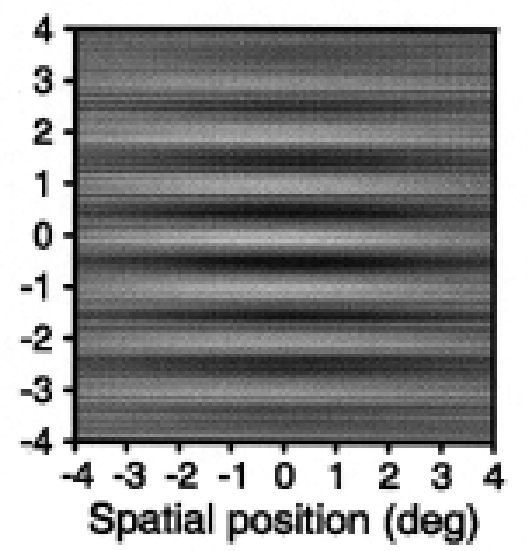

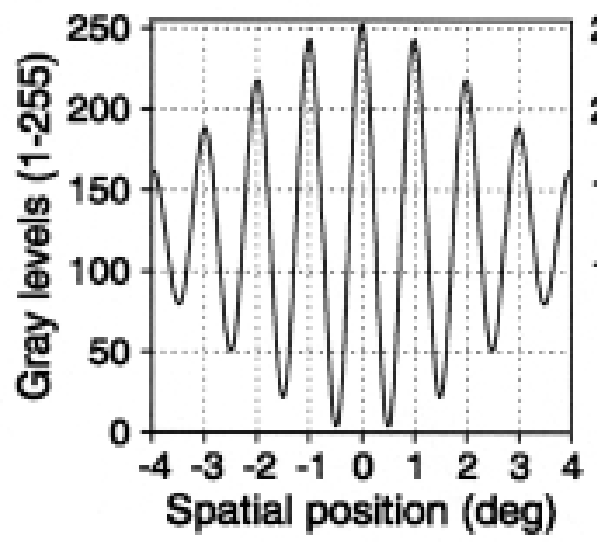

a

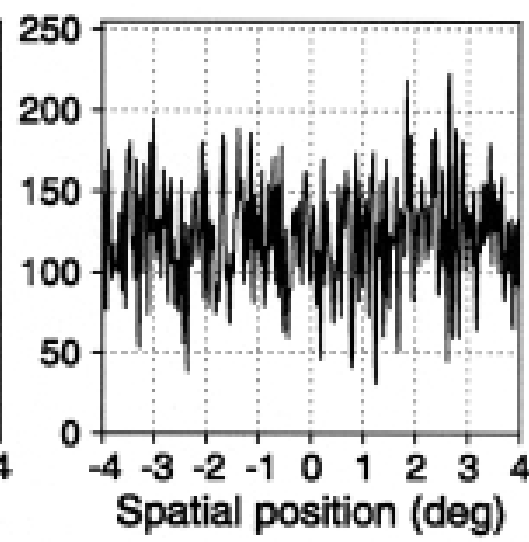

b

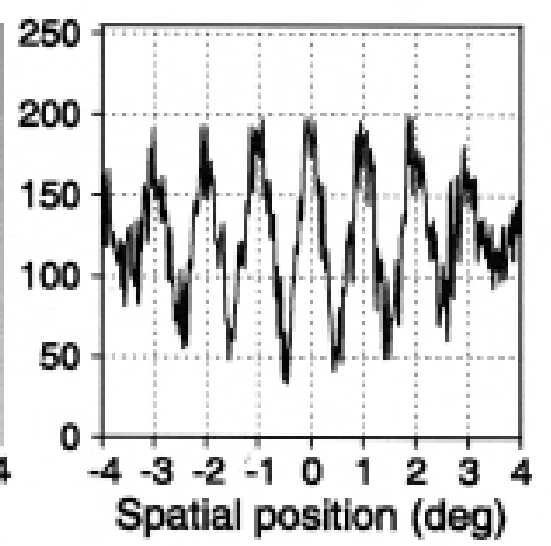

c

Figure 2. Masking procedure using the frame interleaving method. (a) Odd frame. (b) Even frame. (c) Result of frame interleaving procedure. Upper row: odd frame (test) plus even frame (mask) results in the interleaved stimulus. Lower row: gray level profiles along the vertical axis. Note that the stimulus contrast was halved.

The visual stimulus after interleaving is the desired stimulus (i.e., the combination of both) but with its frame rate and contrasts effectively halved (image of Figure $2 \mathrm{c}$ ). (The effective frame rate of $60 \mathrm{~Hz}$ is above flicker fusion frequency). Thus, the effective contrast of test and mask is half of their contrast set in the LUT (Schofield \& Georgeson). In this paper, the effects of interleaving were corrected by software in such a way that $m_{M A S K}$ and $m_{\text {TEST }}$ were as expected.

White noise masks were displayed with one of five power spectral density levels, $N_{0}=0.002 \times 10^{-3} \times 4^{k}(\mathrm{c} / \mathrm{deg})^{-1}, k=$ $0, \ldots, 4)$. Usually, the noise level has been manipulated by varying the standard deviation $(\sigma)$ of the noise luminance distribution (Thomas, 1985), at the expense of reducing the range of gray levels of digital images. Our intention was not to modify the digital image in framestore. Therefore, the noise power level was controlled with the LUT of VSG by setting $m_{\text {MASK }}$ in such a way as to obtain the root-mean-squared contrast, $c_{R M S}$, which corresponds to the desired level, that is,

$$
m_{M A S K}=\frac{I_{0} c_{R M S}}{\sqrt{\mathrm{a}-\mathrm{b}^{2}}+c_{R M S}\left(I_{0}-b\right)}, \quad 0<m_{M A S K}<1,
$$

(Sierra-Vázquez \& Serrano-Pedraza, 2006), where $I_{0}=128$, $c_{R M S}=\sqrt{2 W N_{0}}, a=\sum \sum I_{M A S K}^{2}(i, j) /(N R \times N C)$ and $b=\sum \sum$ $I_{M A S K}(i, j) /(N R \times N C)$, where $I_{M A S K}$ is the digital image of masker noise. Interleaving procedure limits the noise samples that may be used in the experiment: If a noise mask sample needed a $m_{M A S K}$ greater than 0.5 to obtain the desired level, this sample was rejected and another sample with an $m_{\text {MASK }}$ lower than 0.5 was used.

\section{Procedure}

The experiment was carried out in a quiet, dark room. The only light source was the monitor screen. Subjects sat $105 \mathrm{~cm}$ away from the screen. A chin-rest was used to stabilize the subject's head and to control observation distance. CDTs of the test masked by noise were measured 
using an adaptive Bayesian staircase in a two-interval, two-alternative forced-choice (2I, 2AFC) task. Each trial started with a fixation cross presented at the center of the screen for $500 \mathrm{~ms}$ and was divided into two presentation intervals of $500 \mathrm{~ms}$ with a $500-\mathrm{ms}$ interval between the two presentations. During each observation interval, a tone sounded to indicate that the stimulus was being presented. During each presentation interval, stimuli were displayed using a temporal Gaussian envelope with a standard deviation of $100 \mathrm{~ms}$, and truncated to obtain the overall duration of $500 \mathrm{~ms}$. One interval contained the mask plus the test; the other contained the mask stimulus alone. The observer's task was to indicate which interval contained the test signal. In practice, mask and test were presented in both intervals using the interleave procedure, according to Equation 1: in one interval, test contrast was set to zero, that is, mean luminance, and noise mask was presented with its corresponding contrast; in the other interval, both test and mask with their corresponding contrasts were presented. In order to prevent learning, a different stochastic noise sample was used in the two presentation intervals in each trial. The noise samples used in each trial were chosen at random from the previously generated set of 140 images. At the end of each trial, the observer indicated which presentation interval contained the test by pressing a key on the computer keyboard. A new trial was initiated only after the observer's response, thus the experiment proceeded at a pace determined by the observer.

In the experiment, 30 conditions (combination of 6 spatial frequencies and 5 power spectral densities) were tested and the corresponding CDTs were obtained. In each experimental session, a spatial frequency was fixed; five Bayesian staircases were run separately in parallel and randomly interwoven, each with one of the five noise levels. In each staircase, the $m_{\text {MASK }}$ of noise samples was held at the required noise level. For the fixed spatial frequency and noise level, the contrast of test grating (i.e., $m_{\text {TEST }}$ ) was varied from trial to trial according to the Bayesian staircase. The configuration of the Bayesian staircases was as follows: prior probability density function was uniform; model likelihood function, $M$, was symmetric (logistic function) about the threshold (Emerson, 1986; Madigan \& Williams, 1987) and defined as

$$
\mathrm{M}(x ; U)=\gamma+\frac{1-\lambda-\gamma}{1+\exp [\beta(\mathrm{a}+x-U)]},
$$

where $U$ is the log contrast of stimulus and the parameters are:

$$
\beta=\frac{2}{\sigma} \ln \left[\frac{1-\lambda-\delta}{\delta}\right], \alpha=\frac{2}{\beta} \ln \left[\frac{1-\lambda-\pi}{\pi-\gamma}\right]
$$

(both defined by García-Pérez, 1998, Appendix A with different notation), $\lambda=0.01, \gamma=0.5, \pi=0.75, \delta=0.01$, $\sigma=0.8$ (this value is a compromise between an empirical result of Watson and Pelli, 1983, and the theoretical recommendations of Alcalá-Quintana and García-Pérez, 2004); the value of the contrast in each trial was obtained from the mean of the posterior probability density function (Emerson, 1986; King-Smith, Grigsby, Vingrys, Benes \& Supowit, 1994); the threshold was estimated from the mean of the final probability density function (Emerson; Madigan $\&$ Williams). The stopping rule for the staircases was the number of trials (70 trials) (Emerson; Madigan \& Williams). The configuration used here for the Bayesian staircases followed Alcalá-Quintana and García-Pérez (2004). The value of the contrast threshold corresponds to the value 0.75 of the subject psychometric function. This value was taken as the CDT, $m\left(u_{0} ; N_{0}\right)$, for the tested noise masker level $\left(N_{0}\right)$ and the spatial frequency $\left(u_{0}\right)$. Each staircase lasted 5 minutes approximately and each experimental session, 25 minutes.

Previously, CDTs of Gabor patches with no noise or absolute thresholds, $m_{0}$, were obtained with the abovementioned procedure but with $m_{\text {MASK }}$ set to zero.

\section{Fitting the Model to Data}

Contrast sensitivity (CS) was fitted with Equation 2. It must be clear that the CBM model only has three free parameters: $s, b$ and $B$. Once the masked CDTs are known, it is possible to estimate parameters $b$ and $B$. Regrettably, it is not possible to obtain $s$ from data of white noise masking because there are infinite pairs $(b, B)$ that fit the data, one for each value of $s$. Although the value of $s$ affects the value of $B_{o c t}$, fortunately, it does not affect the shape of its variation with peak frequency. Because $s$ is anchored to a value with other types of noise, the value of parameter $s$ for each observer (see Table 1) was obtained from previous experiments of masking in which we used low-pass, high-pass, band-pass, double band-pass, and notched mask noises, and with the same two observers and experimental conditions (Serrano-Pedraza, 2005). Taking this clarification into account, theoretical thresholds from the CBM model for white noise were fitted simultaneously using Equation 6 to experimental data at all noise levels for the supposed two bandwidth regimens (constant and variable bandwidth). To apply Equation 6, we can ignore the Gaussian window of grating stimuli because, as shown in Appendix A, the power contrast of the Gabor patches is constant when the window has a fixed size and for spatial frequencies above $0.1 \mathrm{c} / \mathrm{deg}$ (Figure A, thick line). Best-fitting values of parameters $A$ and $a$ of Equation 2, $b$ and $B$ of Equation 4, and $b$ of Equation 5 were obtained using a least-squares fitting procedure. The sum of squared errors between squared values of empirical and theoretical CDTs was minimized using the Nelder-Mead simplex search method (Nelder \& Mead, 1965). 


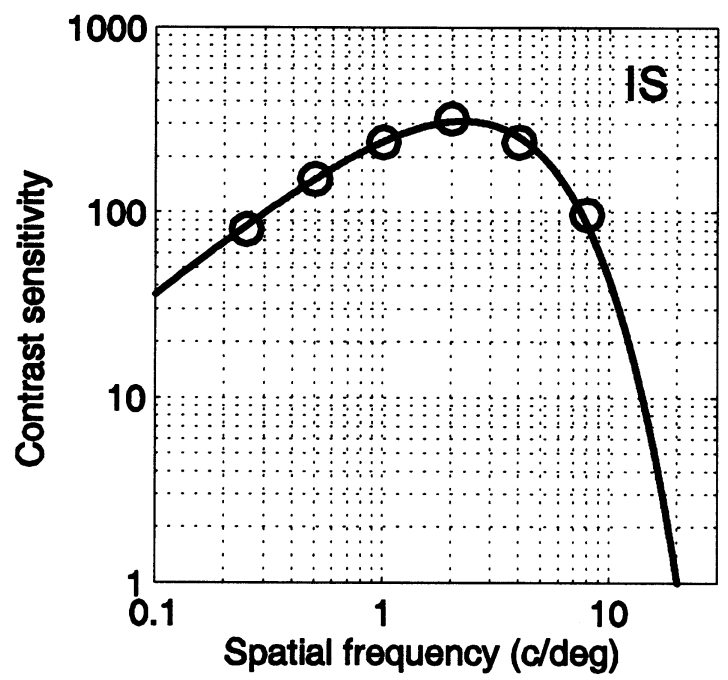

a

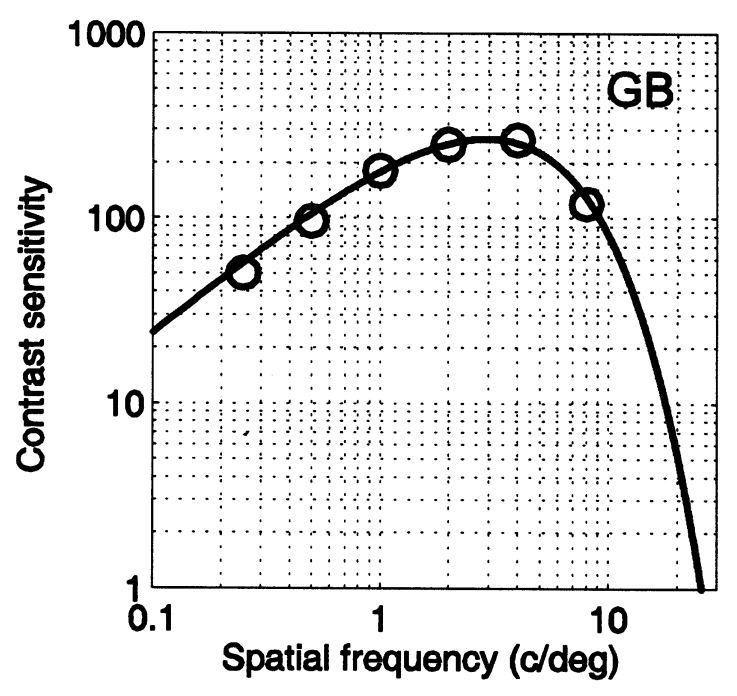

b

Figure 3. Contrast sensitivity (open circles) for Gabor patches without external noise for observers IS (a) and GB (b). The solid curve in each panel shows the CSF calculated in Equation 2. Best-fitting values for the parameter of the CSFs are: Observer IS: $A=377.323$, $a=0.4455$. Observer GB: $A=249.76, a=0.3424$. Extrapolated visual acuities are $20 \mathrm{c} / \mathrm{deg}$ (observer IS) and $22 \mathrm{c} / \mathrm{deg}$ (observer GB) at the luminance level of $15 \mathrm{~cd} / \mathrm{m}^{2}$.

\section{Results}

Figure 3 shows the contrast sensitivity (open circles) to Gabor patches without external noise for the six spatial frequencies for the two observers (Figure $3 a$ and $3 b$ ). The solid line in each panel shows the CSF result of fitting data to Equation 2 (parameter estimates are in the Figure caption). The CSF plotted on a log-log scale has the typical inverted$\mathrm{U}$ shape with a fall-out of sensitivity both at low and at high spatial frequencies (compare with Robson, 1966, at a mean luminance of $20 \mathrm{~cd} / \mathrm{m}^{2}$, temporal frequency of $1 \mathrm{~Hz}$, and fixed field size). Both observers have normal (extrapolated) visual acuity given the mean luminance level used (Hess \& Nordby, 1986, their Figure 6, subject with normal vision).

The results of the masking experiment for the two observers are plotted on a log-log scale in Figure $4 a$ and replotted in Figure 4c. In Figure 4a, the squared CDTs of masked grating (filled symbols) are plotted as a function of the spatial frequency of the grating signal for each of the five noise levels. The thick solid line is the squared absolute threshold curve (obtained using data from Figure 3). The effect of increasing noise power spectral density in CDT is to raise the threshold (i.e., to reduce the sensitivity) not only for high spatial frequencies but also for low spatial frequencies, and, at the same time, to equate them in log-log scale at high levels. The panel of Figure 4a also shows the fitted CDTs (open symbols), and theoretical (squared) threshold curves (thin lines) results of fitting the model with variable $B_{o c t}$ to experimental data for both observers. The fitted masking patterns agree with data when filter bandwidth decreases with peak spatial frequency (see the log RMSE for both observers inserted in panels). The most striking feature of theoretical curves is their progressive leveling-off to become almost flat at the high noise level used. The best-fitting estimates of parameter are shown in Table 1 and the relative bandwidth (in octaves) as a function of peak spatial frequency of filters is plotted for the two observers on a linear scale in Figure $4 \mathrm{~b}$. Figure $4 \mathrm{c}$ shows the fitted CDT result of forcing $B_{\text {oct }}$ to be constant and its estimated values are plotted in Figure 4d. The poor fit to data with constant $B_{o c t}$ is easily observed.

Table 1

Values of Variable Octave Bandwidth Parameters

\begin{tabular}{cccccc}
\hline \multirow{2}{*}{ Observ } & \multicolumn{2}{c}{ Detection model } & \multicolumn{2}{c}{ Parameter (Equation 4) } & \multirow{2}{*}{ RMS error of fits (log units) } \\
\cline { 2 - 5 } & Type of channel & $s^{*}$ & $B$ & $b$ & -3.1264 \\
\hline IS & Fixed & 0.35 & 4.4985 & 0.6822 & -2.9715 \\
\hline
\end{tabular}

Note. ${ }^{*}$ Values from previous masking experiments (Serrano-Pedraza, 2005) 

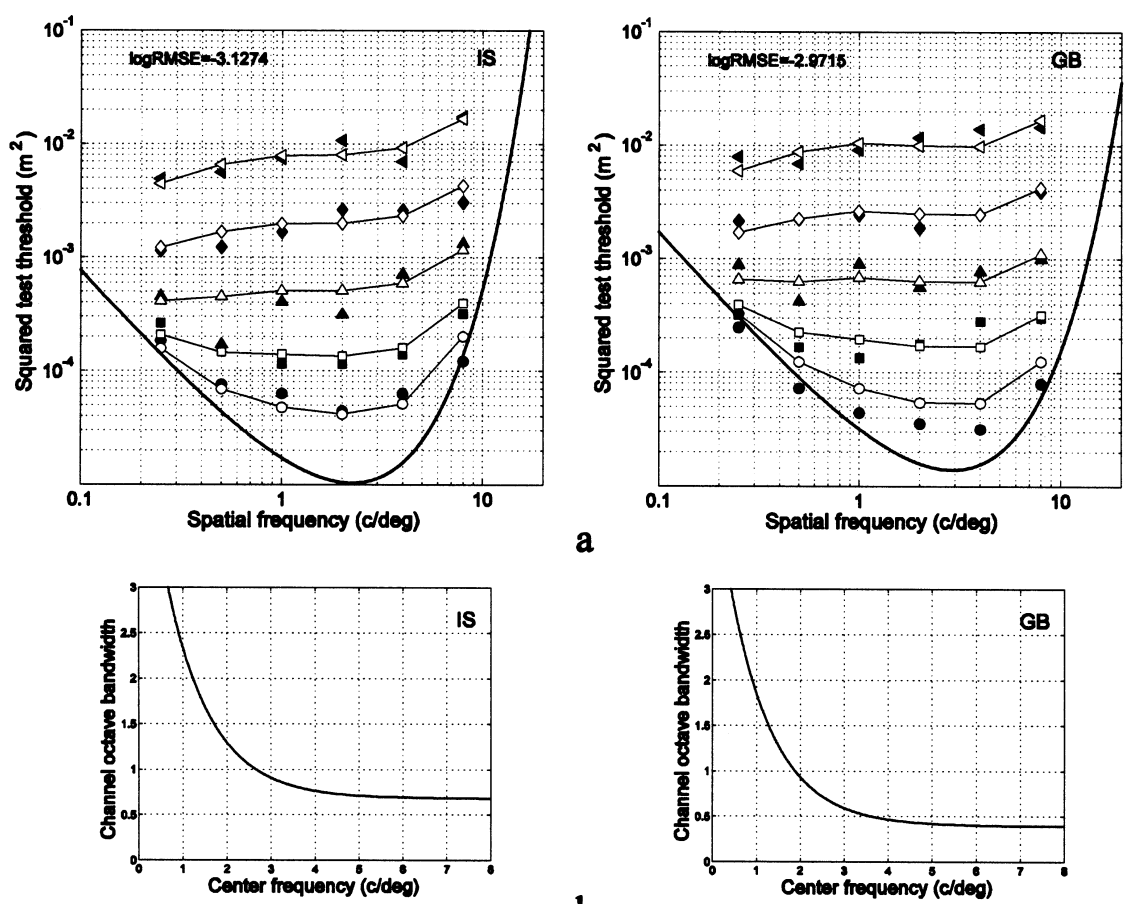

b
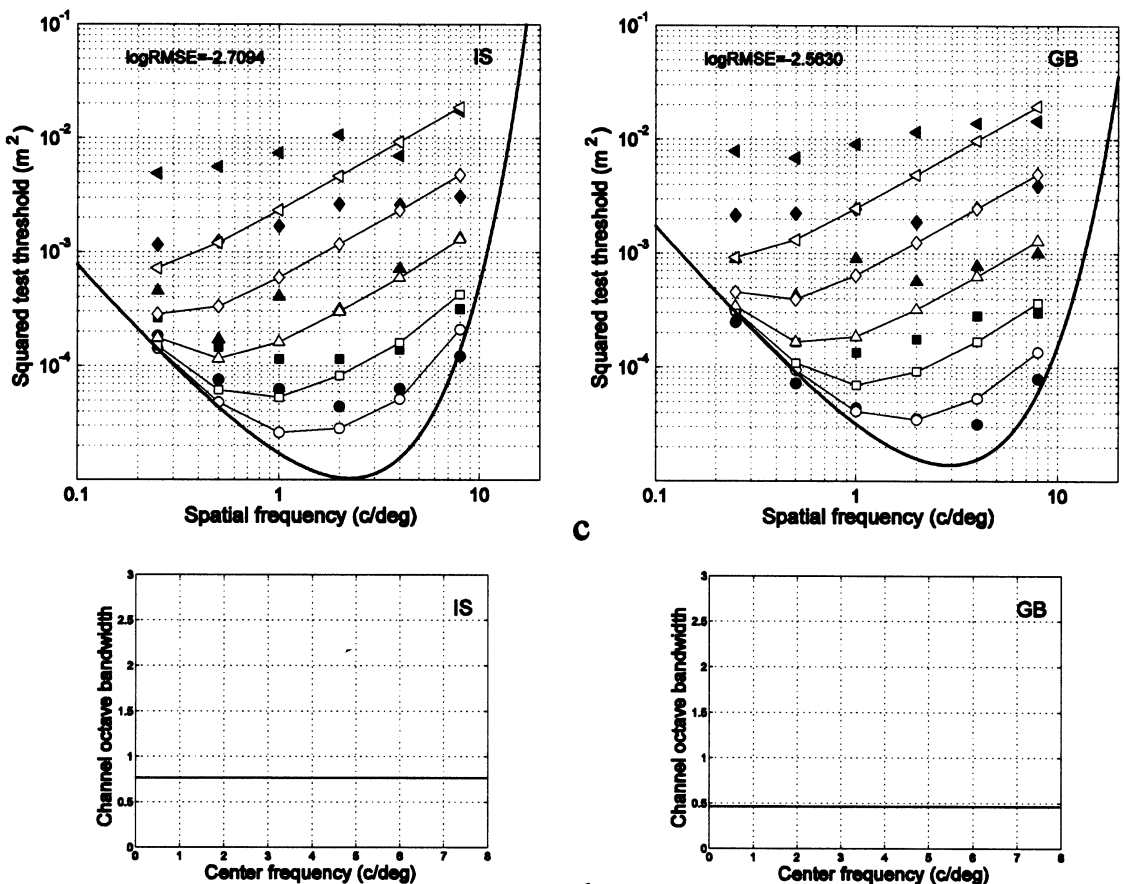

d

Figure 4. Thresholds for detection of Gabor patches in the presence of white noise of different levels and fits of the critical-band-masking model for observers IS and GB. (a) Data and fit assuming variable octave bandwidth. In each panel, experimental squared CDTs for test (filled symbols) and fitted squared CDTs from the critical-band-masking model (open symbols) are plotted on a log-log scale as a function of the spatial frequency of Gabor patch for each noise level. Symbols for CDTs at each noise level, increasing from bottom to top, are: circles, squares, triangles pointing up, diamonds, and triangles pointing left. The specific values can be obtained from $N_{0}=$ $0.002 \times 10^{-3} \times 4^{\mathrm{k}}(\mathrm{c} / \mathrm{deg})^{-1}, k=0, \ldots, 4$. Solid lines connecting open symbols are the model threshold curves; the heavy line is the absolute threshold curve. (b) Filter bandwidth (full bandwidth at half height, in octaves) results of the best fit as a function of peak spatial frequency (parameter estimates for each observer are in Table 1). (c) Fits assuming constant octave bandwidth. In each panel, replotted experimental data (filled symbols) and fitted squared CDTs from the critical-band-masking model (open symbols) are shown. Symbols of noise levels are as in (a). (d) Filter bandwidth (full bandwidth at half height, in octaves) results of the best fit. 

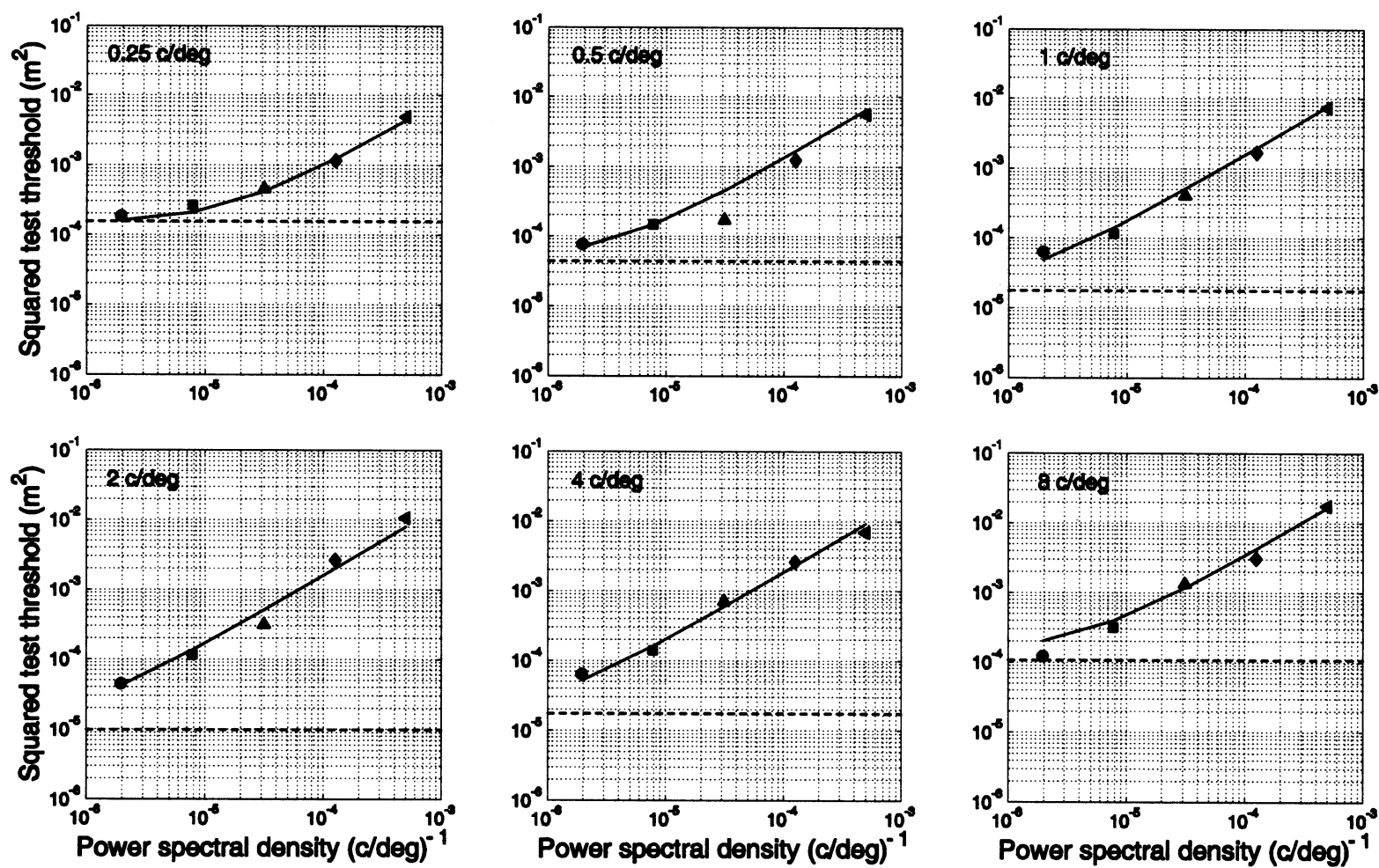

a
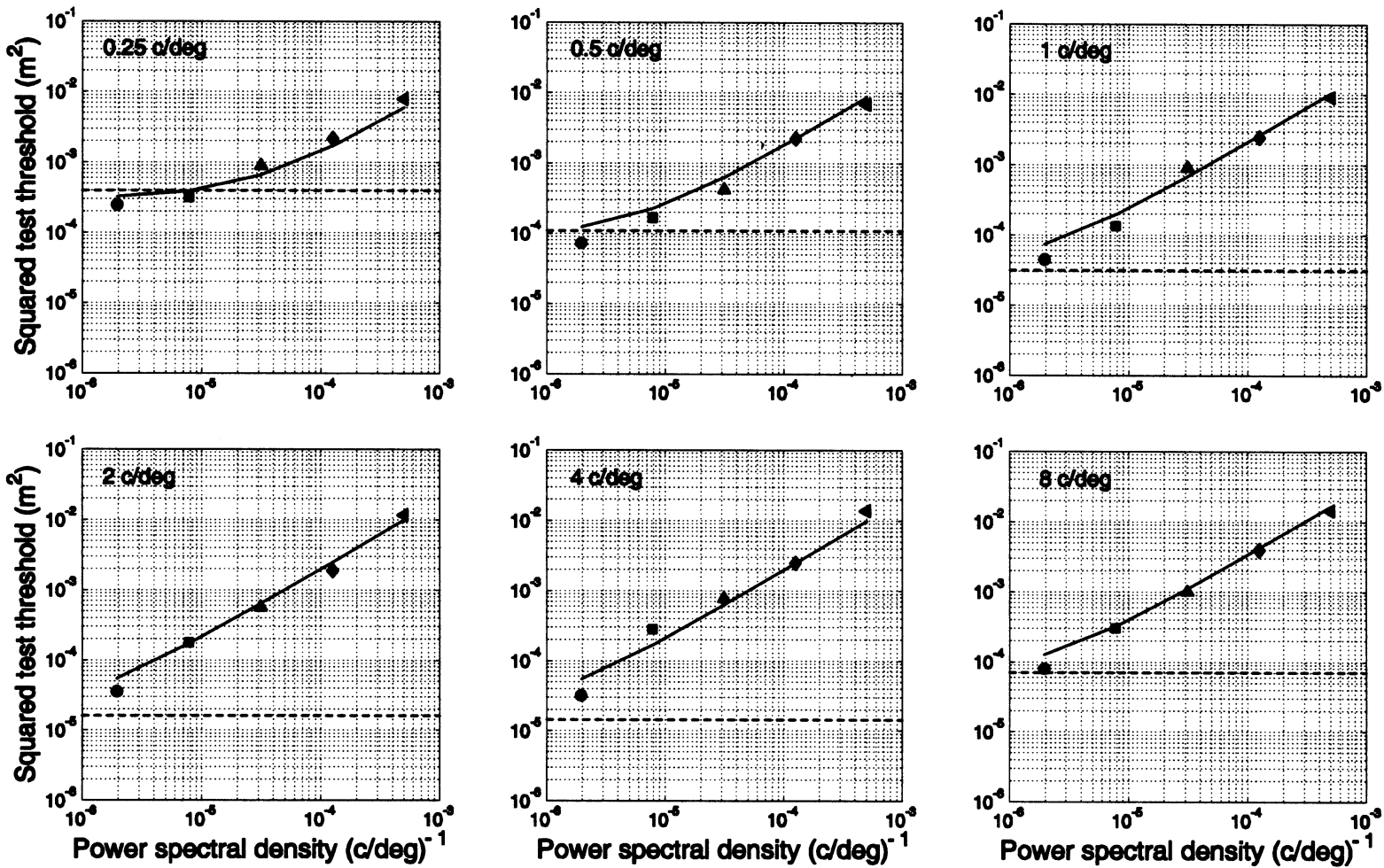

Figure 5. Test of linear relationship between masked (squared) CDTs and noise levels. Each panel shows experimental squared CDT (filled symbols) as a function of noise level for each spatial frequency of Gabor patches and fitted curve (solid line) from Equation 8 , taking into account the best-fitting parameter values in the variable octave bandwidth condition (Table 1). Dashed horizontal lines represent the squared CDT without external noise $\left(m_{0}^{2}\right)$ for the corresponding spatial frequency. (a) Observer IS. (b) Observer GB. 
To test the linear relationship between masked (squared) CDT and noise level predicted by the CBM model, the squared CDT of masked gratings (filled symbols) are replotted in Figure $5 \mathrm{a}$ and $5 \mathrm{~b}$ on a $\log -\log$ scale as a function of the noise power spectral density for each of the six spatial frequencies. For the sake of clarity, we have plotted the data in different panels. In each panel, the dashed line represents the absolute threshold for the corresponding spatial frequency. As can be seen from raw data, squared CDTs rise monotonically with noise level for each spatial frequency, except for an initial decrease at low noise levels for gratings of $0.25 \mathrm{c} / \mathrm{deg}$ and $0.5 \mathrm{c} / \mathrm{deg}$ (subject GB) (possibly due to threshold bias). The solid line through the symbols in each panel is the prediction from Equation 8 for each noise level, taking into account the supposed values of $s$ and the best-fitting values of $b$ and $B$ for variable filter bandwidth (Table 1). As can be seen, predictions from CBM model fit well the experimental data for both observers.

\section{Discussion}

This paper presents data on the changes in threshold curves for sinusoidal grating masked by white noise of different spectral power densities. We applied the CBM model to explain the change in CDTs with noise level and tested two particular shapes of the relationship between relative bandwidth (in octave) and peak frequency of filters. Squared CDTs at different noise levels were fitted to the CBM model and, as a result, the fit was adequate only when visual filters involved in the detection of masked gratings had decreasing octave bandwidth with peak frequency.

Quantitative predictions about masked CDTs need the (squared) absolute threshold as an index of power of internal noise (i.e., as a limit of the observer performance in the absence of external noise). It is known that the CSF shape lacks low spatial frequency attenuation when Gabor patch window scales with spatial frequency (Peli, Arend, Young, \& Goldstein, 1993). This is the shape of the CSF obtained and used by Solomon (2000). Nevertheless, here, the CSFs of the two observers (Figure 3) have the typical shape for CSF when Gabor patches with a fixed size window are used (Barten, 1999), thus it is coherent with the stimulus configuration used in our experiment.

The comparison of empirical and theoretical CDTs shows that the CBM model with variable filter bandwidth fits masking data well (Figures 4a, 4c, and 5), and the fitted threshold detection curves show a progressive flattening with the increase in noise level. Patterns of masking are qualitatively similar to those reported in the literature at the level used in each study. The change in curvature of threshold curves at medium noise levels is qualitatively similar to the effects that Blackwell (1998, his Figure 2) found with 2D static log-Gaussian noise and fixed circular aperture. The flat CDT curve at its highest level is similar to that of Rovamo et al. (1992, his Figure 2) with $2 \mathrm{D}$ white noise and variable aperture. Comparisons must be qualitative because Rovamo et al. and Blackwell use 2D noise and represent CDT or CS (and not squared CDTs) versus spatial frequency. Masked CDTs lower than absolute detection threshold, such as those we found for 0.25 and $0.5 \mathrm{c} / \mathrm{deg}$, were also found by other authors (Losada \& Mullen, 1995; Serrano-Pedraza \& SierraVázquez, 2004). These results have been interpreted as small facilitatory effects of white noise at low levels which are not predicted by the (linear) CBM model and reported for the first time in Blackwell (1998) as due to visual stochastic resonance. Indeed, considering the large size of the standard errors of threshold estimates from Bayesian staircases (Alcalá-Quintana \& García-Pérez, 2004), those data points are so minimally below the respective absolute threshold that they could be considered within the threshold bias.

There is much disagreement in the spatial vision literature over specific bandwidth values. In fact, there are significant variations in the bandwidth estimates of visual filters because direct estimates depend on experimental paradigms (Kelly \& Burbeck, 1984), and derived estimates depend on the assumptions of interpretative theoretical models (Olzak \& Thomas, 1986). This problem is further complicated here by the fact that specific estimates depend on the assumed value of the sensitivity parameter $s$. Therefore, we do not deal with bandwidth filter values, although our estimates are within the usual range (Olzak \& Thomas, Table 7.1). Fortunately, the value of parameter $s$ does not affect the form of the relationship. This fact allows us to discuss our results on filter bandwidth variation with the peak frequency. A number of direct (Henning et al., 1981; Losada \& Mullen, 1995; Stromeyer \& Julesz, 1972) and theoretically derived (Pelli, 1981; Solomon \& Pelli, 1994; Talgar, Pelli, \& Carrasco, 2004) measures indicate that linear bandwidth is roughly proportional to the filter peak frequency, which proves constant octave bandwidth. In addition, constant octave bandwidth has been used to explain the results of white noise masking (Blackwell, 1998; Rovamo et al., 1992). On the contrary, quantitative analysis of our experimental data by means of the CBM model supports the view that the visual filter octave bandwidth decreases with its peak frequency. This statement agrees with direct and derived measures obtained with different types of masker (Schofield \& Georgeson, 2003; Serrano-Pedraza, 2005; Wilson et al., 1983, their Figure 11) as shown in Figure 6. To reconcile these conclusions, it has to be noted that octave bandwidth is almost constant for spatial frequencies above $2.5 \mathrm{c} / \mathrm{deg}$ for both observers (Figure 4b). Therefore, if only higher spatial frequencies were used, bandwidth would appear constant. 
We have obtained slightly different results from those of Solomon (2000) (see Figure 6), even though his curve is shifted down to overlap with ours. In the Appendix, we show that the contrast power of a Gabor patch depends both on its Michelson contrast and its window size. In the case of a Gabor patch whose Gaussian window scales with its spatial frequency (Solomon), contrast power decreases dramatically with window size (i.e., with the spatial frequency of grating) (Figure A, thin line). Thus, the contrast power of scaled Gabor patches is originally affected by a quantity that should be taken into account in calculations. It is important to note that Equation 6 is for the CDTs of gratings without a spatial window. Therefore, it is not possible to apply Equation 6 to fit the CDTs obtained using Gabor patches with scaled windows without further consequences for the estimated bandwidth. On the contrary, if the window size is fixed, as is the case in this paper, the contrast power of the stimuli for spatial frequencies greater than $0.1 \mathrm{c} / \mathrm{deg}$ is affected by a constant factor (Figure A of Appendix, thick line) and it only depends on its Michelson contrast, as required by Equation 6. This fact enables us to use this equation directly, with no further consequences for bandwidth estimates. Thus, the proper application of CBM model supports the non-constant bandwidth version of the visual filterbank.

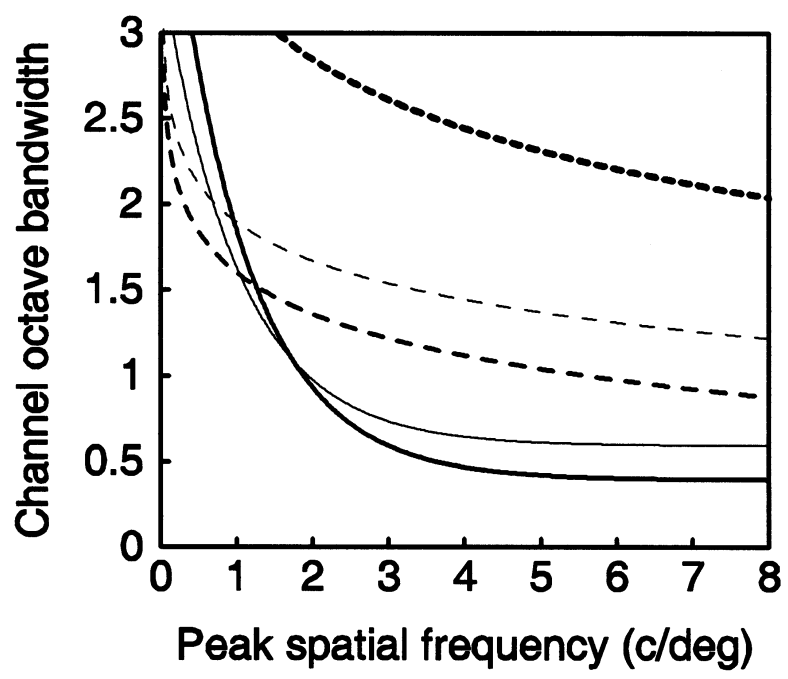

Figure 6. Comparisons of estimates of octave bandwidth as a function of filter peak spatial frequency. Dashed heavy line, estimated function by Solomon (2000) (his Equation 4 with parameter values in his Table 1, fixed-channels detection model); the dashed thin line represents $B_{f}$ in Equation A.5 of Schofield and Georgeson (2003); the dashed thick line represents $B_{f}$ in Equation A.6 of Schofield and Georgeson (2003); the thin solid lines represents $B_{\text {oct }}$ in Equation 4.21 of Serrano-Pedraza (2005) (parameter values of Table 7.3 for observer GB, asymmetric gain function, and fixed-channels detection model); the thick solid line, result from Figure $4 b$ (observer GB).

\section{References}

Alcalá-Quintana, R., \& García-Pérez, M. A. (2004). The role of parametric assumptions in adaptive Bayesian estimation. Psychological Methods, 9, 250-271.

Barten, P.G.J. (1999). Contrast sensitivity of the human eye and its effects on image quality. Bellingham, Washington: SPIE Optical Engineering Press.

Blackwell, K.T. (1998). The effect of white and filtered noise on contrast detection thresholds. Vision Research, 38, 267-280.

DePalma, J. J., \& Lowry, E.M. (1962). Sine-wave response of the visual system. II. Sine-wave and square-wave sensitivity. Journal of the Optical Society of America, 52, 328-335.

De Valois, R.L., Albrecht, D.G., \& Thorell, L.G. (1982). Spatial frequency selectivity of cells in macaque visual cortex. Vision Research, 22, 545-559.

De Valois, R.L., \& De Valois, K.K. (1988). Spatial vision. Oxford: Oxford University Press.

Emerson, P.L. (1986). Observations on maximum-likelihood and Bayesian methods of forced-choice sequential threshold estimation. Perception \& Psychophysics, 39, 151-153.

Fletcher, H. (1940). Auditory patterns. Reviews of Modern Physics, 12, 47-65.

García-Pérez, M.A. (1998). Forced-choice staircases with fixed steps sizes: Asymptotic and small-sample properties. Vision Research, 38, 1861-1881.

García-Pérez, M. A., \& Peli, E. (2001). Luminance artifacts of cathode-ray tube displays for vision research. Spatial Vision, 14, 201-215.

Green, D.M., \& Swets, J.A. (1966). Signal detection theory and psychophysics. Huntington, NY: Krieger.

Hartmann, W.M. (1998). Signals, sound, and sensation. NY: Springer-Verlag.

Henning, G.B., Hertz, B.G., \& Hinton, J.L. (1981). Effects of different hypothetical detection mechanisms on the shape of spatialfrequency filters inferred from masking experiments. I. noise masks. Journal of the Optical Society of America, 71, 574-581.

Hess, R.F., \& Nordby, K. (1986). Spatial and temporal limits of vision in the achromat. Journal of Physiology, 371, 365-385.

Kelly, D.H. (1975). Spatial frequency selectivity in the retina. Vision Research, 15, 665-672.

Kelly, D.H., \& Burbeck, C.A. (1984). Critical problems in spatial vision. CRC Critical Reviews in Biomedical Engineering, 10, 125-177.

King-Smith, P.E., Grigsby, S.S., Vingrys, A.J., Benes, S.C., \& Supowit, A. (1994). Efficient and unbiased modifications of the QUEST threshold method: Theory, simulations, experimental evaluation and practical implementation. Vision Research, 34, 885-912.

Legge, G.E., Kersten, D., \& Burgess, A.E. (1987). Contrast discrimination in noise. Journal of the Optical Society of America A, 4, 391-404.

Losada, M.A., \& Mullen K.T. (1995). Color and luminance spatial tuning estimated by noise masking in the absence of offfrequency looking. Journal of the Optical Society of America A, 12, 250-260. 
Madigan, R., \& Williams, D. (1987). Maximum-likelihood psychometric procedures in two-alternative forced-choice: Evaluation and recommendations. Perception \& Psychophysics, 42, 240-249.

Majaj, N.J., Pelli, D.G., Kurshan, P., \& Palomares, M. (2002). The role of spatial frequency channels in letter identification. Vision Research, 42, 1165-1184.

Moore, B.C.J. (1997). An introduction to the psychology of hearing ( $4^{\text {th }}$ ed.). New York: Academic Press.

Morrone, M.C., \& Burr, D.C. (1988). Feature detection in human vision: A phase-dependent energy model. Proceedings of the Royal Society of London B, 235, 221-245.

Nelder, J.A., \& Mead, R. (1965). A simplex method for function minimization. The Computer Journal, 7, 308-313.

Olzak, L.A., \& Thomas, J.P. (1986). Seeing spatial patterns. In K.R. Boff, L. Kaufman, \& J.P. Thomas (Eds.), Handbook of perception and human performance. Volume I (pp. 7:1-7:56). New York: Wiley.

Patterson, R.D. (1974). Auditory filter shape. Journal of the Acoustical Society of America, 55, 802-809.

Patterson, R.D. (1976). Auditory filter shapes derived with noise stimuli. Journal of the Acoustical Society of America, 59, 640654.

Peli, E., Arend, L., Young, G., \& Goldstein, R. (1993). Contrast sensitivity to patch stimuli: Effects of spatial bandwidth and temporal presentation. Spatial Vision, 7, 1-14.

Pelli, D.G. (1981). Effects of visual noise. Doctoral dissertation. Cambridge University, Cambridge, UK (unpublished).

Pelli, D.G. (1990). The quantum efficiency of vision. In C. Blakemore (Ed.), Vision: Coding and efficiency (pp. 3-24). Cambridge, UK: Cambridge University Press.

Robson, J.G. (1966). Spatial and temporal contrast-sensitivity functions of the visual system. Journal of the Optical Society of America, 56, 1141-1142.

Rovamo, J., Franssila, R., \& Näsänen, R. (1992). Contrast sensitivity as a function of spatial frequency, viewing distance and eccentricity with and without spatial noise. Vision Research, 32, 631-637.

Schofield, A. (1998). Calibration issues in monochrome raster scan displays. Perception and Cognition Tutorial. Birmingham, UK: University of Birmingham.

Schofield, A., \& Georgeson, M.A. (1999). Sensitivity to modulations of luminance and contrast in visual white noise: Separate mechanisms with similar behaviour. Vision Research, 39, 26972716.
Schofield, A., \& Georgeson, M.A. (2003). Sensitivity to contrast modulation: The spatial frequency dependence of second-order vision. Vision Research, 43, 243-259.

Serrano-Pedraza, I. (2005). Procesos visuales de demodulación espacial. Unpublished doctoral dissertation, Universidad Complutense, Madrid, Spain. Available at www.ucm.es/BUCM/tesis/psi/ucm-t28909.pdf.

Serrano-Pedraza, I., \& Sierra-Vázquez, V. (2004). Efecto del ruido binario en la detección de enrejados sinusoidales. Programa y Resúmenes. Madrid: SEPEX (abstract 141).

Serrano-Pedraza I., \& Sierra-Vázquez, V. (2005). The effect of white-noise mask level on sinewave detection thresholds. Perception (Suppl.), 34, 102 (abstract).

Serrano-Pedraza, I., \& Sierra-Vázquez, V. (2006). El paradigma de enmascaramiento con ruido visual: simulación del modelo basado en bandas críticas. In M.J. Contreras, J. Botella, R. Cabestrero, \& B. Gil-Gómez (Eds.), Lecturas de psicología experimental (pp. 183-192). Madrid: UNED.

Sierra-Vázquez, V., \& Serrano-Pedraza, I. (2006). An algorithm to equate the contrast power of filtered natural images. Manuscript submitted for publication.

Solomon, J.A. (2000). Channel selection with non-white-noise masks. Journal of the Optical Society of America A, 17, 986-993.

Solomon, J.A., \& Pelli, D.G. (1994). The visual filter mediating letter identification. Nature, 369, 395-397.

Stromeyer III, C.F., \& Julesz, B. (1972). Spatial-frequency masking in vision: Critical bands and spread of masking. Journal of the Optical Society of America, 62, 1221-1232.

Talgar, C.P., Pelli, D.G., \& Carrasco, M. (2004). Cover attention enhances letter identification without affecting channel tuning. Journal of Vision, 2, 22-31.

Thomas, J.P. (1985). Effect of static-noise and grating masks on detection and identification of grating targets. Journal of the Optical Society of America A, 2, 1586-1592.

Van Nes, F.L., \& Bouman, M.A. (1967). Spatial modulation transfer function of the human eye. Journal of the Optical Society of America, 57, 401-406.

Watson, A.B., \& Pelli, D.G. (1983). QUEST: A Bayesian adaptive psychometric method. Perception and Psychophysics, 33, 113-120.

Wilson, H.R., McFarlane, D.K., \& Phillips, G.C. (1983). Spatial frequency tuning of orientation selective units estimated by oblique masking. Vision Research, 23, 873-882.

Received January 11, 2006

Review received June 20, 2006 Accepted June 30, 2006 


\section{Appendix}

\section{Numerical evaluation of the contrast power of a Gabor patch}

The contrast power, $P_{C}$, of a $2 \mathrm{D}$ contrast function $f$ is (Pelli, 1990)

$$
P_{\mathrm{C}}=\frac{1}{g_{x} \times g_{y}} \int_{-\mathrm{g} / 2 / 2}^{\mathrm{g} / 2} \int_{-\mathrm{g}, / 2}^{\mathrm{g} / 2} f^{2}(x, y) \mathrm{d} x \mathrm{dy}
$$

where $g_{x}, g_{v}$, in deg, are the spatial dimensions of the contrast function. Let $f$ be the Gabor patch defined by Equation 10 with Michelson contrast $m$. Its contrast power is

$$
P_{\mathrm{C}}=\frac{m^{2}}{2} \times\left[\frac{2}{g_{x} \times g_{y}} \int_{-\mathrm{g} / 2 / 2}^{\mathrm{g} / 2} \int_{-\mathrm{g} / 2}^{\mathrm{g} / 2}\left[w^{2}(x, y) \cos ^{2}\left(2 \pi u_{0} \mathrm{y}\right)\right] \mathrm{d} x \mathrm{dy}\right],
$$

where $m^{2} / 2$ is the contrast power of a sinusoidal grating without windowing (Hartmann, 1998, p. 26) and $w$ is the 2D circular Gaussian window of the Gabor patch. Figure A shows the numerical evaluation of the quantity between square brackets, $K$, as a function of the spatial frequency of Gabor patch in two cases: with fixed window size $\left(g_{x}=g_{y}=8\right.$ deg and $s_{x}=s_{y}$ $=2.5 \mathrm{deg}$ ) (thick line), and with scaled window size $\left(\mathrm{sx}=1 /\left(\sqrt{2} u_{0}\right)\right)$ (thin line). Note that, in the first case, coefficient $K$ is almost constant for spatial frequencies greater than $0.1 \mathrm{c} / \mathrm{deg}$; thus, contrast power of Gabor patch depends only on its contrast $m$. On the contrary, with scaled window size, the coefficient decreases with the spatial frequency to a negligible value; thus, in this case, contrast power depends jointly on the contrast and the spatial frequency of the patch.

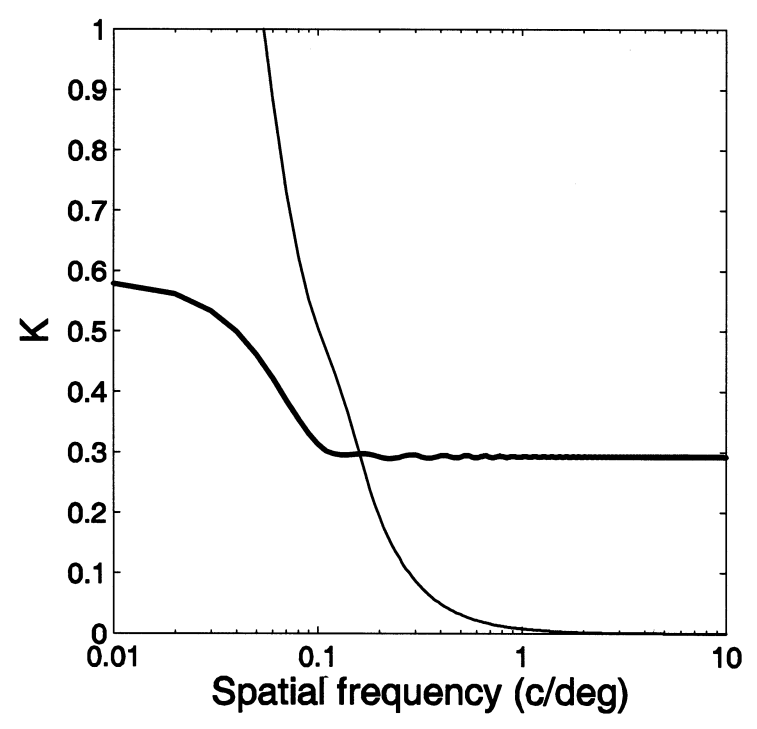

Figure A. Numerical evaluation of factor $K$ in Equation A1 as a function of the spatial frequency of a Gabor patch with a Gaussian window of fixed size (thick line) and when the size of the window scales with the spatial frequency of grating (thin line). 